\title{
Constructing NSSD Molecular Graphs
}

Ivan Gutman, ${ }^{1,2}, *$ Boris Furtula, ${ }^{1}$ Alexander Farrugia, ${ }^{3}$ Irene Sciriha ${ }^{3}$



RECEIVED: September 22, 2016 * REVISED: October 31, 2016 * ACCEPTED: November 3, 2016

THIS PAPER IS DEDICATED TO PROFESSOR NENAD TRINAJSTIĆ ON THE OCCASION OF HIS 80 ${ }^{\text {TH }}$ BIRTHDAY

\begin{abstract}
A graph is said to be non-singular if it has no eigenvalue equal to zero; otherwise it is singular. Molecular graphs that are non-singular and also have the property that all subgraphs of them obtained by deleting a single vertex are themselves singular, known as NSSD graphs, are of importance in the theory of molecular $\pi$-electron conductors; NSSD = non-singular graph with a singular deck. Whereas all non-singular bipartite graphs (therefore, the molecular graphs of all closed-shell alternant conjugated hydrocarbons) are NSSD, the non-bipartite case is much more complicated. Only a limited number of non-bipartite molecular graphs have the NSSD property. Several methods for constructing such molecular graphs are presented.
\end{abstract}

Keywords: Non-singular graph with singular deck, NSSD, molecular graph, single-molecule electron conduction, molecular electronics.

\section{PROLOGUE}

$\mathrm{N}$ the 1970s, Nenad Trinajstić and his research group achieved a breakthrough in theoretical chemistry by applying the mathematical theory of graph spectra to Hückel molecular orbital (HMO) theory. ${ }^{[1-6]}$ Among the first problems studied were the existence and the determination of the number of non-bonding molecular orbitals. ${ }^{[7-10]}$ As time passed, interest in $\mathrm{HMO}$ theory and its graph-theoretical aspects faded, and research along these lines gradually ceased. Recently, however, a new and somewhat unexpected application of $\mathrm{HMO}$ theory emerged. ${ }^{[11-14]}$ In view of this, the results and methods developed in the 1970s, especially those pertaining to nonbonding molecular orbitals, regained importance. It is fortunate that this is happening just in the time when Nenad Trinajstić, our dear Trina, is celebrating his $80^{\text {th }}$ birthday. Congratulations!

\section{INTRODUCTION}

Fowler et al. ${ }^{[11-14]}$ developed a tight-binding model for describing the steady-state current created by the injection of ballistic electrons via external contacts into an unsaturated conjugated $\pi$-electron system, treated as a single-molecule conductor. Within this model, the conjugated molecule is said to be an ipso insulator if it behaves as an insulator for a particular connection via a single $\pi$-electron center. If this is the case with all conjugated centers, then the respective molecule is an ipso omni insulator; for details see Tables IV and IX, as well as Theorem 6.1 in the paper. ${ }^{[14]}$

Let $G$ be the molecular graph of a conjugated $\pi$ electron system, ${ }^{[5,6]}$ and let $\lambda_{1}, \lambda_{2}, \ldots, \lambda_{n}$ be the eigenvalues of its adjacency matrix (usually referred ${ }^{[5,6]}$ to as the eigenvalues of the underlying graph). Recall that such graphs are connected and have vertices whose degrees do not exceed three. ${ }^{[5,6]}$

The number of eigenvalues of the graph $G$ that are equal to zero is called the nullity of $G$, and will be denoted by $\eta(G)$. A (molecular) graph whose nullity is greater than zero is said to be singular. If $\eta(G)=0$, then $G$ is nonsingular.

Denote by $\phi(G, \lambda)$ the characteristic polynomial of the graph $G$. As is well known, ${ }^{[5,6]}$ the graph $G$ is singular if and only if $\phi(G, 0)=0$.

If $v_{1}, v_{2}, \ldots, v_{n}$ are the vertices of the graph $G$, then the collection of its vertex-deleted subgraphs, 
$G-v_{i}, i=1,2, \ldots, n$, is referred to as its deck. ${ }^{[15-19]}$ If the graph $G$ is non-singular, i.e., $\eta(G)=0$, but all elements of its deck are singular, i.e., $\eta\left(G-v_{i}\right)>0, i=1,2, \ldots, n$, then $G$ is "non-singular with a singular deck", or, abbreviated, NSSD. ${ }^{[18]}$ Because of the Cauchy interlacing theorem, ${ }^{[20]}$ it follows that $\eta\left(G-v_{i}\right)=1, i=1,2, \ldots, n$.

NSSD molecular graphs are interesting from a theoretical-chemistry point of view, because any such graph represents an ipso omni insulator, and vice-versa: the molecular graph of any conjugated $\pi$-electron system that is ipso omni insulator is NSSD. The proof of this result is found in Theorem 4.4 of Ref. 14 (necessity) and in Deductions 5.7 and 5.9 of the same paper.

In this paper, we examine the structure of NSSD molecular graphs and ways how these can be constructed. It is easy to show that the molecular graph of any alternant conjugated hydrocarbon without non-bonding molecular orbitals is an NSSD graph. Contrary to this, among molecular graphs of non-alternant conjugated hydrocarbons, a surprisingly small number has the NSSD property. Our main contribution is the construction of nonbipartite NDDS molecular graphs.

\section{BIPARTITE NSSD MOLECULAR GRAPHS}

At this point, we recall that a graph is said to be bipartite if it does not possess odd-membered cycles. Bipartite molecular graphs represent alternant conjugated $\pi$-electron systems. For such graphs, the classic Coulson-Rushbrooke pairing theorem holds, ${ }^{[5,6,21]}$ according to which the eigenvalues are paired as $\lambda_{n-i+1}=-\lambda_{i}, i=1,2, \ldots, n$. Consequently, if the number of vertices $n$ is odd, then at least one eigenvalue is equal to zero and the respective graph is singular.

This has the following straightforward and elementary consequence:

Proposition 1a. A bipartite graph is NSSD if and only if it is non-singular. There are no bipartite NSSD graphs with an odd number of vertices.

Within the Hückel (tight-binding) molecular orbital approximation, each zero eigenvalue of the molecular graph corresponds to a non-bonding molecular orbital (NBMO). ${ }^{[5,6]}$ The absence of NBMOs is one of the necessary (but not sufficient) conditions for stability of the underlying conjugated $\pi$-electron system. ${ }^{[10]}$ Thus, the chemical consequence of Proposition 1a is:

Proposition 1b. A bipartite molecular graph is NSSD if it represents a stable alternant conjugated $\pi$-electron system with no NBMOs. Such graphs necessarily possess an even number of vertices.

According to Proposition 1b, each stable alternant conjugated hydrocarbon is associated with an NSSD graph

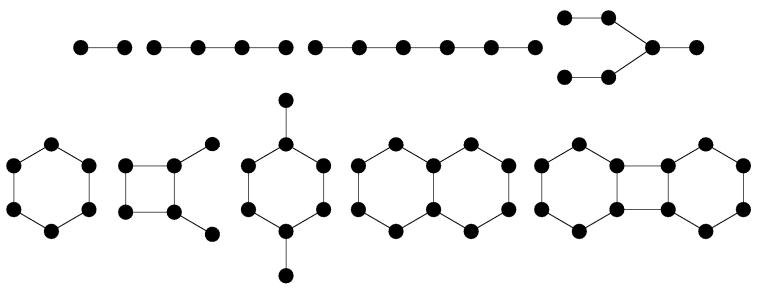

Figure 1. Molecular graphs of some typical alternant hydrocarbons that have no non-bonding molecular orbitals. These all are NSSD graphs.

and is therefore (within the Fowler model) an ipso omni insulator. Some characteristic examples are given in Figure 1. These coincide with the examples found in the paper by Fowler et al. ${ }^{[14]}$

\section{NON-BIPARTITE NSSD MOLECULAR GRAPHS}

Whereas the molecular graphs of all stable, closed-shell alternant hydrocarbons are NSSD, the situation with nonbipartite species is significantly different. Non-bipartite NSSD graphs happen to be relatively rare, and this especially applies to molecular graphs.

First of all, the data in Table 1 show that the vast majority of connected graphs are non-bipartite.

We have performed a systematic computer search for $n$-vertex non-bipartite NSSD graphs and molecular graphs, for all values of $n$ up to $n=11$. The data in Table 1 reveal that in the case $n=11$, more than one billion graphs had to be tested. For this reason, such calculations for $n>11$ would be technically non-feasible. Results of similar kind, but restricted to a few classes of molecular graphs, were earlier reported by Fowler et al. ${ }^{[14]}$ Our findings fully agree with these, and corroborate them.

Our findings for an odd number of vertices are the following:

For $n=3,5,7,9$, there are no non-bipartite NSSD graphs.

For $n=11$, there are exactly 15 non-bipartite NSSD graphs, depicted in Figure 2. None of these is a molecular graph.

Our findings for an even number of vertices are the following:

For $n=4$, there are no non-bipartite NSSD graphs.

For $n=6$, there exists a single non-bipartite NSSD molecular graph, the [3]radialene graph $G_{1}$ in Figure 3.

For $n=8$, there exist exactly four non-bipartite NSSD molecular graphs (see Figure 3 ).

For $n=10$, out of over one million non-bipartite graphs, only some two dozen NSSD molecular graphs have been discovered. Their main representatives are depicted in Figure 4. 
Table 1. Number of connected $n$-vertex graphs

\begin{tabular}{lccc}
\hline$n$ & all connected & bipartite & non-bipartite \\
\hline 4 & 6 & 3 & 3 \\
5 & 21 & 5 & 16 \\
6 & 112 & 17 & 95 \\
7 & 853 & 44 & 809 \\
8 & 11117 & 182 & 10935 \\
9 & 261080 & 730 & 260350 \\
10 & 11716571 & 4032 & 11712539 \\
11 & 1006700565 & 25598 & 1006674967 \\
12 & 164059830476 & 212780 & 164059617696 \\
13 & 50335907869219 & 2241730 & 50335905627489 \\
14 & 29003487462848061 & 31193324 & 29003487431654737 \\
15 & 31397381142761241960 & 575252112 & 31397381142185989848 \\
\hline
\end{tabular}



Figure 2. Among 1006674967 non-bipartite graphs with 11 vertices, only 15 are NSSD. None of these are molecular graphs.

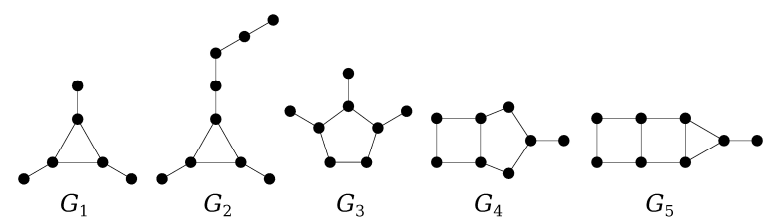

Figure 3. The non-bipartite molecular NSSD graphs with 6 and 8 vertices. Note that $G_{2}$ and $G_{3}$ are constructed from $G_{1}$ by means of Transformation 2 and 4, respectively.

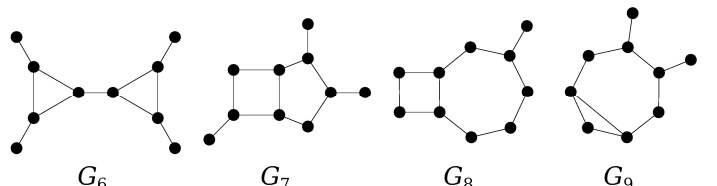

$G_{7}$
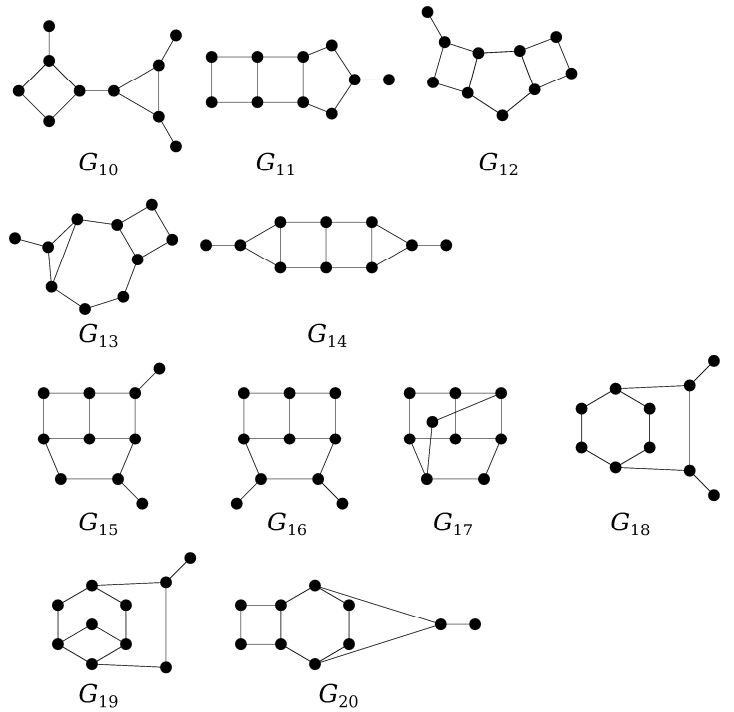

Figure 4. Some non-bipartite molecular NSSD graphs with 10 vertices. The species constructed by Transformation 1 (namely,[5]radialene) and those constructed by making use of Transformation 2 are not depicted (but see Figure 6). The graph $G_{6}$ is constructed by Transformation 3 (cf. Figure 7). The vertex degrees of the NSSD graphs $G_{15}, G_{16}, G_{17}$, $G_{18}, G_{19}, G_{20}$ do not exceed 3, but for chemical reasons these should not be considered as representing real conjugated $\pi$ electron systems. 

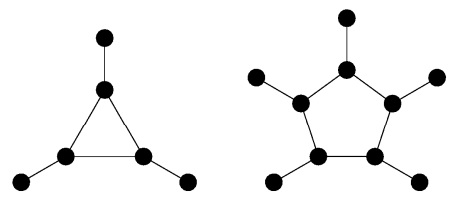

$G_{1}$

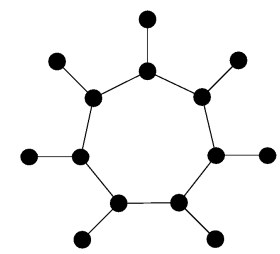

Figure 5. The three smallest non-bipartite NSSD molecular graphs constructed using Transformation 1.

\section{CONSTRUCTING NON-BIPARTITE NSSD GRAPHS}

In this section, we describe a few general methods for constructing non-bipartite NSSD graphs.

Transformation 1. Let $G$ be any graph with $n$ vertices. Let $G^{*}$ be a graph with $2 n$ vertices, obtained by attaching a new pendent vertex to each vertex of $G$. Then $G^{*}$ is an NSSD graph. Proof. If $u$ is a pendent vertex of a graph $H$, adjacent to the vertex $v$, then ${ }^{[8,10]}$

$$
\eta(H)=\eta(H-u-v)
$$

Applying Eq. (1) repeatedly to all but one pendent vertices of $G^{*}$, we end with the 2-vertex connected graph whose nullity is zero. Therefore, $\eta\left(G^{*}\right)=0$. If $u$ is any pendent vertex of $G^{*}$, then by applying Eq.(1) repeatedly to all pendent vertices of $G^{*}-u$, we end with the single-vertex graph whose nullity is one. Therefore, $\eta\left(G^{*}-u\right)=1$. If $v$ is any non-pendent vertex of $G^{*}$, then the subgraph $G^{*}-v$ contains an isolated vertex and is therefore singular. Thus, $G^{*}$ is non-singular, and all its vertex-deleted subgraphs are singular, i.e., $G^{*}$ is an NSSD graph. $\square$

The only non-bipartite NSSD molecular graphs that can be obtained by means of Transformation 1 are the radialene graphs, see Figure 5.

Let $G_{A}$ be a graph and let be $x$ one of its vertices. Let $G_{B}$ be another graph and $y$ one of its vertices. Denote by $G_{A}(x, y) G_{B}$ the graph obtained from $G_{A}$ and $G_{B}$ by joining the vertices $x$ and $y$ by a new edge.

Transformation 2. If $G_{A}$ and $G_{B}$ are two NSSD graphs, and $x$ and $y$ any two of their vertices, then $G_{A}(x, y) G_{B}$ is also an NSSD graph.

Proof. The characteristic polynomial of $G_{A}(x, y) G_{B}$ conforms to the relation ${ }^{[22,23]}$

$\mathbf{f}\left(G_{A}(x, y) G_{B}, \lambda\right)=\mathbf{f}\left(G_{A}, \lambda\right) f\left(G_{B}, \lambda\right)-\mathbf{f}\left(G_{A}-x, \lambda\right) f\left(G_{B}-y, \lambda\right)$.

Since $G_{A}$ and $G_{B}$ are assumed to be NSSD, $\phi\left(G_{A}, 0\right) \neq 0$, $\phi\left(G_{B}, 0\right) \neq 0$ whereas $\phi\left(G_{A}-x, 0\right)=0, \phi\left(G_{B}-y, 0\right)=0$, implying that $\phi\left(G_{A}(x, y) G_{B}, 0\right) \neq 0$. Thus, $G_{A}(x, y) G_{B}$ is nonsingular.

The subgraph $G_{A}(x, y) G_{B}-x$ is singular because one of its components is $G_{A}-x$, which is singular since $G_{A}$ is assumed to be NSSD. By the same reasoning, $G_{A}(x, y) G_{B}-y$ is also singular.

Let $z$ be any vertex of $G_{A}(x, y) G_{B}$, different from $x$ and $y$. Without loss of generality, assume that $z$ is a vertex of $G_{\mathrm{A}}$.

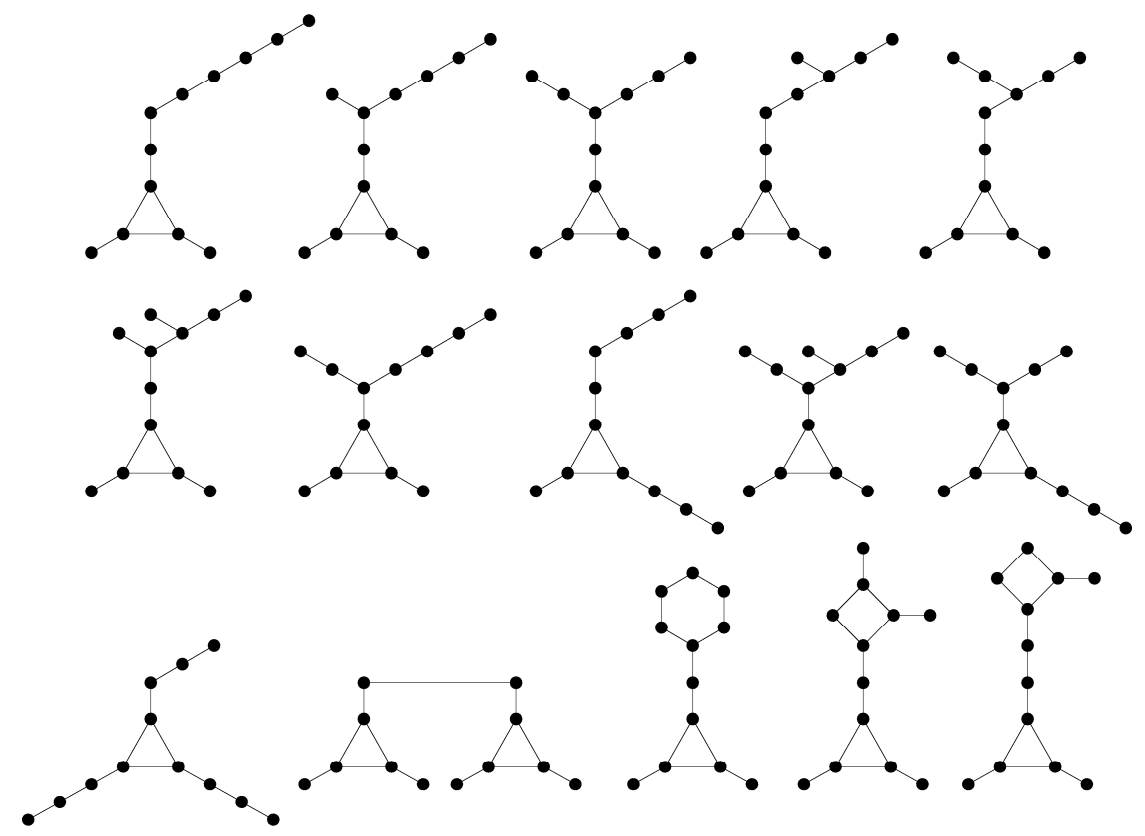

Figure 6. All 12-vertex molecular graphs constructed by means of Transformation 2, starting from the [3]radialene graph $G_{1}$ (cf. Figure 2). 

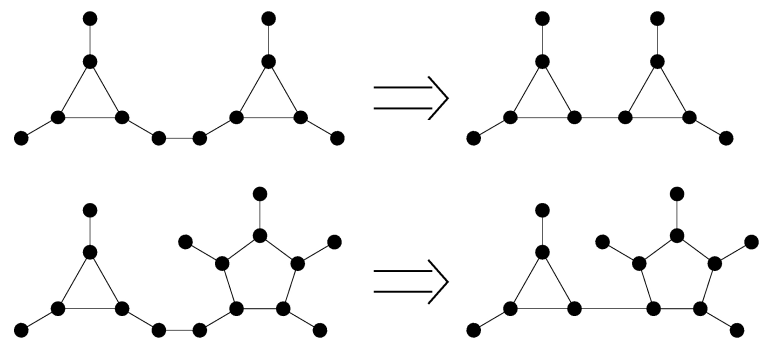

Figure 7. Applying Transformation 3 to construct new nonbipartite NSSD molecular graphs from those obtained by means of Transformation 2.

Then

$\phi\left(G_{A}(x, y) G_{B}-z, \lambda\right)=$

$$
\phi\left(G_{\mathrm{A}}-z, \lambda\right) \phi\left(G_{\mathrm{B}}, \lambda\right)-\phi\left(G_{\mathrm{A}}-z-x, \lambda\right) \phi\left(G_{\mathrm{B}}-y, \lambda\right) .
$$

It follows that $\phi\left(G_{\mathrm{A}}(x, y) G_{\mathrm{B}}-z, 0\right)=0$ since $\phi\left(G_{\mathrm{A}}-z, 0\right)=0$ and $\phi\left(G_{B}-y, 0\right)=0$. Therefore, $G_{A}(x, y) G_{B}-z$ is singular for any choice of the vertex z. Thus, $G_{A}(x, y) G_{B}$ is a nonsingular graph with a singular deck.

A few examples illustrating Transformation 2 are depicted in Figure 6.

Transformation 3. Let $G_{A}(x, y) G_{B}$ be the same graph as in Transformation 2, such that its vertices $x$ and $y$ are both of degree two. Let $x$ be adjacent to $x^{\prime}$ and $y$, whereas $y$ be adjacent to $x$ and $y^{\prime}$. Construct the graph $G_{A}(-) G_{B}$ by deleting from $G_{A}(x, y) G_{B}$ the vertices $x$ and $y$ and then joining the vertices $x^{\prime}$ and $y^{\prime}$ by a new edge. If $G_{A}(x, y) G_{B}$ is NSSD, then $G_{A}(-) G_{B}$ is also NSSD.

The proof is analogous to the proof of Transformation 2 and is omitted.

Two examples illustrating the Transformation 3 are shown in Figure 7.

Transformation 4. An NSSD graph is obtained by applying the reverse of Transformation 3 to any edge of a nonbipartite unicyclic NSSD graph, i.e., by inserting two new degree-two vertices on an edge. Illustrative examples are provided in Figure 8.

\section{DISCUSSION AND CONCLUDING REMARKS}

Our findings indicate that non-bipartite NSSD graphs and molecular graphs are relatively rare objects. Yet, by means of Transformations 1-4, infinitely many molecular graphs of this kind can be constructed. It is worth noting that all our construction methods produce graphs having an even number of vertices.

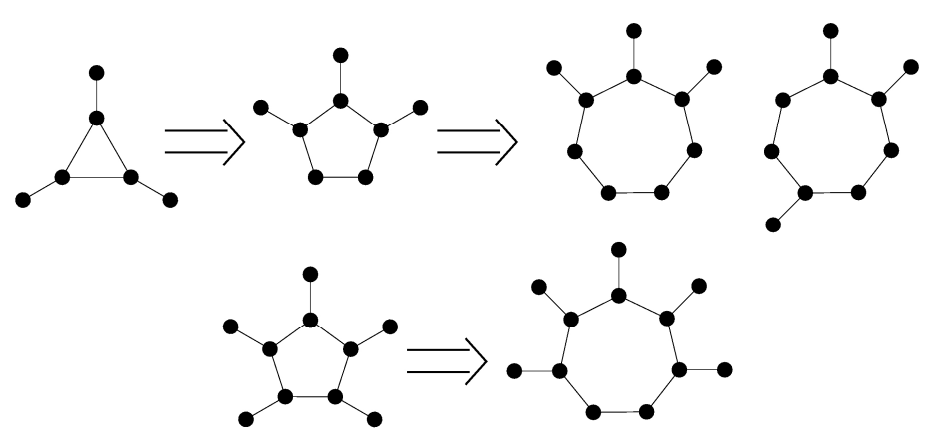

Figure 8. Applying Transformation 4 to construct new non-bipartite NSSD molecular graphs from those obtained by means of Transformation 1.

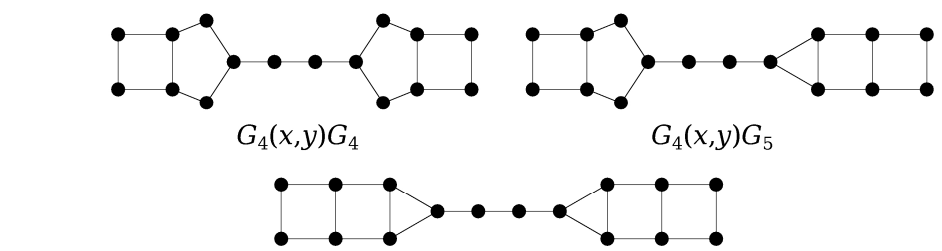

$G_{5}(x, y) G_{5}$



$$
G_{4}(-) G_{4} \quad G_{4}(-) G_{5} \quad G_{5}(-) G_{5}
$$

Figure 9. NSSD molecular graphs without pendent vertices, constructed by means of Transformations 2 and 3 from the graphs $G_{4}$ and $G_{5}$ from Figure 3 . 
Bearing this in mind, recalling that bipartite oddvertex NSSD graphs cannot exist at all, and that not a single non-bipartite molecular graph of this kind was detected up to $n=11$, we are inclined to conjecture the following: Conjecture. There are no NSSD molecular graphs with an odd number of vertices.

By inspecting the examples depicted in Figures 3-8, one notices that these all contain pendent vertices (vertices of degree one). This, however, is not a generally valid regularity. Figure 9 shows NSSD molecular graphs with 14 and 16 vertices, without pendent vertices. These seem to be the smallest examples of this kind.

Thus, there exist NSSD molecular graphs whose vertex degree are all two or three. Whether there are such graphs with all vertices of degree three, perhaps fullerene graphs, remains a problem to be solved in the future.

Acknowledgment. The second author was partially supported by the Serbian Ministry of Science and Education, through the Grant No. 174033.

\section{REFERENCES}

[1] A. Graovac, I. Gutman, N. Trinajstić, T. Živković, Theor. Chim. Acta 1972, 26, 67.

[2] I. Gutman, N. Trinajstić, Topics Curr. Chem. 1973, 42, 49.

[3] N. Trinajstić, Hückel theory and topology, in Semiempirical Methods of Electronic Structure Calculation. Part A: Techniques (Ed.: G. A. Segal), Plenum Press, New York, 1977, pp. 1-27.

[4] N. Trinajstić, Int. J. Quantum Chem. Quantum Chem. Symp. 1977, 11, 469.

[5] A. Graovac, I. Gutman, N. Trinajstić, Topological Approach to the Theory of Conjugated Molecules, Springer, Berlin, 1977.
[6] N. Trinajstić, Chemical Graph Theory, CRC Press, Boca Raton, 1983; 2nd revised ed. 1992.

[7] T. Živković, Croat. Chem. Acta 1972, 44, 351.

[8] D. Cvetković, I. Gutman, N. Trinajstić, Croat. Chem. Acta 1972, 44, 365.

[9] D. Cvetković, I. Gutman, N. Trinajstić, Theor. Chim. Acta 1974, 34, 129.

[10] D. Cvetković, I. Gutman, N. Trinajstić, J. Mol. Struct. 1975, 28, 289.

[11] B. T. Pickup and P. W. Fowler, Chem. Phys. Lett. 2008, 459, 196.

[12] P. W. Fowler, B. T. Pickup, T. Z. Todorova, Chem. Phys. Lett. 2008, 465, 142.

[13] P. W. Fowler, B. T. Pickup, T. Z. Todorova, W. A. Myrvold, J. Chem. Phys. 2009, 131, 044104.

[14] P. W. Fowler, B. T. Pickup, T. Z. Todorova, M. Borg, I. Sciriha, J. Chem. Phys. 2014, 140, 054115.

[15] S. M. Ulam, A Collection of Mathematical Problems, Wiley, New York, 1960.

[16] F. Harary, Graph Theory, Addison-Wesley, Reading, 1969.

[17] I. Sciriha, Lin. Algebra Appl. 2009, 430, 78.

[18] A. Farrugia, J.B. Gauci, and I. Sciriha, Special Matrices 2013, 1, 28.

[19] A. Farrugia, J. B. Gauci, and I. Sciriha, Discr. Appl. Math. 2016, 202, 5.

[20] I. Gutman and N. Trinajstić, J. Chem. Soc. Faraday Trans. 1977, I/ 73, 435.

[21] C. A. Coulson, G. S. Rushbrooke. Proc. Cambridge Phil. Soc. 1940, 46, 193.

[22] I. Gutman, O. E. Polansky, Theor. Chim. Acta 1981, 60, 203.

[23] I. Gutman, O. E. Polansky, Mathematical Concepts in Organic Chemistry, Springer, Berlin, 1986. 